\title{
Novas orientações da Direção-Geral de Saúde para a diabetes gestacional: uma apreciação crítica
}

Carla Gouveia, ${ }^{1}$ Mónica Granja, ${ }^{2}$ Armando Brito de Sá, ${ }^{3}$ Luís Filipe Gomes, ${ }^{4}$ José Augusto Simões, ${ }^{5}$ Rosa Gallego ${ }^{6}$

\section{RESUMO}

As novas orientações técnicas da Direção-Geral de Saúde (DGS) para a diabetes gestacional (DG) alteram os procedimentos ligados ao rastreio, acompanhamento e terapêutica desta patologia. Discute-se a base científica das referidas normas e as suas implicações nos ganhos em saúde, custos, recursos de saúde e impacto na nova população de grávidas afetadas.

O diagnóstico de DG baseado numa glicemia em jejum superior a $92 \mathrm{mg} / \mathrm{dL}$ na primeira consulta da gravidez não se encontra suportado por nenhuma das referências citadas na norma da DGS.

O diagnóstico de DG utilizando os novos valores limiares na determinação da prova de tolerância à glicose realizada entre as 24 e as 28 semanas de gestação multiplica cerca de dez vezes a prevalência de diabetes gestacional, aumentando a necessidade de recursos de saúde para o seu acompanhamento.

Encontra-se estabelecido por meta-análises que o rastreio universal de grávidas não tem vantagens em relação ao rastreio seletivo a grávidas com fatores de risco. Os estudos de custo-benefício são favoráveis ao rastreio seletivo comparativamente ao rastreio universal (grau de recomendação $B$ e nível de evidência 3A).

Não se encontra estabelecido que a intervenção terapêutica sobre estas novas gravidezes patológicas seja capaz de evitar a morbilidade perinatal prevista pelo estudo HAPO e não foram estudados os efeitos adversos que esta terapêutica possa originar.

Será prudente suspender esta norma e regressar ao modelo anterior de rastreio e diagnóstico de DG até que evidência robusta recomende a sua modificação.

Palavras-chave: Diabetes Gestacional; Rastreio; Diagnóstico; Abordagem da Doença; Normas.

\section{INTRODUÇÃO}

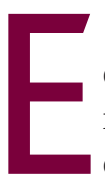
m janeiro de 2011, a Direção-Geral de Saúde (DGS) emitiu um conjunto de novas orientações técnicas relacionadas com a diabetes. Uma dessas orientações, «Terapêutica da Diabetes Mellitus tipo 2: metformina», ${ }^{1}$ foi objeto de análise recente. ${ }^{2}$

1Médica de família, Unidade de Saúde Familiar LoureSaudável, Centro de Saúde de Loures, Lisboa

${ }^{2}$ Médica de família, Centro de Saúde da Senhora da Hora, Matosinhos

${ }^{3}$ Médico de família, Unidade de Saúde Familiar Rodrigues Miguéis, Lisboa; Professor do Instituto de Medicina Preventiva da Faculdade de Medicina da Universidade de Lisboa ${ }^{4}$ Médico de família, Centro de Saúde de Lagoa; Professor Auxiliar Convidado do Mestrado Integrado de Medicina da Universidade do Algarve

${ }^{5}$ Médico de família, Unidade de Saúde Familiar Marquês de Marialva, Cantanhede; Professor da Escola Superior de Saúde da Universidade de Aveiro

'Médica de família, Unidade de Cuidados de Saúde Personalizados de Vila Franca de Xira; Núcleo de Diabetes da Associação Portuguesa de Medicina Geral e Familiar
Foram igualmente publicados pela DGS dois documentos no âmbito da diabetes gestacional (DG), «Diagnóstico e conduta na Diabetes Gestacional $»^{3}$ e «Relatório de Consenso sobre Diabetes e Gravidez», ${ }^{4}$ determinando uma nova estratégia de rastreio da DG. Existem modificações importantes na estratégia preconizada em relação ao anteriormente proposto, nomeadamente no que diz respeito ao teste de rastreio utilizado e ao momento da sua aplicação, bem como a uma alteração dos valores de referência utilizados como critérios de diagnóstico e de introdução da terapêutica com insulina. O acompanhamento das grávidas com DG identificada continua a realizar-se, como anteriormente, na consulta de medicina materno-fetal hospitalar, mas a reclassificação pós-parto passa a ser responsabilidade destas unidades e não do médico de 
família, como anteriormente.

Apresenta-se, neste artigo, uma visão crítica dos documentos científicos que servem de base à referida orientação técnica e discutem-se eventuais benefícios, riscos e custos da sua aplicação.

\section{MOMENTO DE APLICAÇÃO DO TESTE DE RASTREIO}

A orientação técnica «Diagnóstico e conduta na Diabetes Gestacional ${ }^{3}$ define dois momentos de aplicação do teste de rastreio: a primeira consulta e o período entre as 24 e as 28 semanas de gestação.

Não existe, no entanto, evidência que suporte a utilização do limite de $92 \mathrm{mg} / \mathrm{dL}$ de glicemia em jejum no diagnóstico de DG na primeira consulta. Este valor foi obtido pelo grupo de peritos da Associação Internacional de Diabetes $^{5}$ (AID) com base nos resultados do estudo HAPO. ${ }^{6}$ Nesse estudo foi demonstrado aumento do risco de eventos perinatais adversos (que incluem tanto eventos maternos como do recém-nascido) relacionados com o aumento dos valores de glicemia das grávidas entre as 24 e as 32 semanas e não em idades gestacionais inferiores (grau de recomendação B, nível de evidência 2B).*A American Diabetes Association (ADA), na sua declaração de posição de 2012, não adopta nenhum critério de diagnóstico de DG na primeira consulta (grau de recomendação B e nível de evidência 2B).?

A segunda determinação, entre as 24 e as 28 semanas, é justificada por ocorrer suficientemente cedo para que o início da terapêutica ainda possa alterar o aparecimento dos eventos perinatais adversos e suficientemente tarde para poder revelar-se alteração do metabolismo glucídico provocada pela gravidez. ${ }^{8-12}$

\section{TESTE DE RASTREIO A UTILIZAR NO DIAGNÓSTICO}

A nova orientação técnica define que o teste de rastreio a utilizar para o diagnóstico de DG será a prova de tolerância à glicose realizada com três determinações: em jejum, uma e duas horas após a ingestão de $75 \mathrm{~g}$ de glicose.

Este teste de rastreio foi escolhido pela AID, ${ }^{5}$ dado permitir uniformizar o teste de diagnóstico de DG com o utilizado para o diagnóstico de diabetes na população não

\footnotetext{
* Grau de recomendação e níveis de evidência de acordo com «Classificação de Oxford Centre for Evidence-Based Medicine» disponível em: http://portal.saude.gov.br/portal/arquivos/pdf/tabela_nivel_evidencia.pdf
}

grávida e por ter sido o teste utilizado no estudo HAPO. ${ }^{6}$

Uma meta-análise da Cochrane comparou várias formas de rastreio de $\mathrm{DG},{ }^{13}$ tendo concluído que diferentes testes resultam em diferentes prevalências de DG (grau de recomendação B e nível de evidência 3A). Não foi encontrada evidência convincente mostrando a superioridade de um tipo de teste de rastreio em relação a outro (nomeadamente prova de O'Sullivan em comparação com a prova de tolerância à glicose, quer com $100 \mathrm{~g}$ de glicose quer com $75 \mathrm{~g}$ de glicose); do mesmo modo, não foi demonstrada vantagem em rastrear a população total de grávidas em comparação com as grávidas com fatores de risco para DG.

\section{ALTERAÇÃO DOS VALORES DE REFERÊNCIA}

A alteração dos valores de referência para diagnóstico de DG baseia-se no consenso da AID que, por sua vez, se baseia no estudo HAPO. Este estudo mostra uma associação linear entre os valores de glicemia (tanto em jejum, como na prova de tolerância à glicose com $75 \mathrm{~g}$ entre as 24 e as 32 semanas de gestação) e a probabilidade de ocorrência de eventos perinatais adversos (grau de recomendação B e nível de evidência 2B). Por se tratar de uma associação linear contínua não foi identificado um nível a partir do qual intervir, a AID estabeleceu por consenso valores limite que condicionam uma probabilidade de eventos perinatais adversos 1,75 vezes superior ao risco basal. Para estes valores limite $†$ são previsíveis os seguintes aumentos da probabilidade de eventos perinatais adversos: $5 \%$ de bebés grandes para a idade gestacional, $9 \%$ de cesarianas, $1 \%$ de hipoglicemias neonatais, $6 \%$ de aumento do péptido C no cordão umbilical, $5 \%$ de partos pré-termo, $1 \%$ de distocias de ombro, $5 \%$ de internamentos na unidade de cuidados intensivos neonatais, $5 \%$ de hiperbilirrubinémias e $4 \%$ de pré-eclâmpsias.

Esta alteração de critérios de diagnóstico para DG originou, na população do estudo HAPO, um aumento de prevalência de DG de 1,7\% para 17,8\%. Passa assim a ser classificada como doente uma percentagem importante da população de grávidas anteriormente considerada saudável.

Quase metade das mulheres do estudo HAPO não aceitou participar no mesmo, o que nos remete para questões sobre a representatividade da amostra estudada. As

† Glicemia em jejum de 92 mg/dL, de 180 mg/dL uma hora após a ingestão de $75 \mathrm{~g}$ de glicose e de $153 \mathrm{mg} / \mathrm{dL}$ após duas horas. 
mulheres que participaram tinham idade média superior à habitual, índice de massa corporal (IMC) mais elevado e maior taxa de cesarianas. ${ }^{14}$ Não foi feito ajuste para estas variáveis de confundimento nem para o aumento de peso durante a gravidez mas, em estudos anteriores, ${ }^{15-18} \mathrm{O}$ ajuste para as possíveis variáveis de confundimento (idade materna, índice de massa corporal prévio, aumento de peso durante a gestação e presença de fatores de risco para DG) tornou os resultados estatisticamente não significativos.

Os estudos citados pelo estudo HAPO, que corroboram o aumento de eventos perinatais adversos, foram realizados em grávidas com fatores de risco para DG. A dúvida persiste sobre se o HAPO não terá sofrido um viés de seleção. Pode recomendar-se que mulheres grávidas com idades e IMC prévio semelhantes aos das mulheres incluídas no estudo HAPO tenham como objetivo manter a glicemia tão baixa quanto possível a partir da $24 .{ }^{a}$ semana. No entanto, extrapolar estes resultados para todas as mulheres grávidas, com estas ou outras características, e para a totalidade de tempo de gestação, usando como valor de referência um valor definido por um consenso de peritos, reduz o nível de evidência destas orientações. Não existindo menção, nestas orientações, a estudos controlados aleatorizados, meta-análises ou revisões sistemáticas, não é seguro que os novos valores de referência propostos para o diagnóstico de DG sejam adequados para todas as mulheres grávidas.

O American College of Obstetrics and Gynecology anunciou em 2011 que não recomenda a adoção destes novos valores de referência, visto não estarem demonstradas as vantagens em termos de resultados materno-fetais e se prever que a sua adoção traga um aumento significativo dos custos. ${ }^{19}$

\section{IMPLICAÇÕES PRÁTICAS DA NOVA FORMA DE DIAGNÓSTICO DE DIABETES GESTACIONAL}

De acordo com a atualização do Relatório de Consenso sobre Diabetes e Gravidez (da responsabilidade das Sociedades Portuguesas de Endocrinologia, Diabetes e Metabolismo, de Diabetologia e de Obstetrícia e Medicina Materno-Fetal e da secção de Neonatologia da Sociedade Portuguesa de Pediatria), ${ }^{4}$ após o diagnóstico de DG devem ser instituídas medidas não farmacológicas: plano alimentar personalizado (considerando o valor calórico total e o IMC prévio à gravidez) associado a exercício físico regular (grau de recomendação B e nível de evidência 2B). Caso os ob- jetivos de glicemia-alvo (glicemia em jejum entre 60 e 90 $\mathrm{mg} / \mathrm{dL}$ e glicemia uma hora após a refeição entre 100 e 120 $\mathrm{mg} / \mathrm{dL}$ ) não sejam atingidos no período de uma a duas semanas, deve ser iniciada insulinoterapia (grau de recomendação A e nível de evidência 1B). Às mulheres que apresentem glicemias de jejum entre 100 e $140 \mathrm{mg} / \mathrm{dL}$, após o primeiro trimestre, devem ser instituídos antidiabéticos orais (grau de recomendação B e nível de evidência 2B). As orientações indicam ainda a auto-vigilância da glicemia capilar (grau de recomendação B e nível de evidência 3A). As grávidas sob medidas não farmacológicas devem realizar determinações de glicemia pelo menos quatro vezes por dia (em jejum e uma hora após as refeições principais) enquanto as tratadas com insulina devem realizar pelo menos seis pesquisas diárias (três em jejum e três pós-prandiais), não sendo recomendada frequência especial para as grávidas medicadas com anti-diabéticos orais.

\section{RASTREIO DE DG E EVENTOS PERINATAIS ADVERSOS}

Uma revisão Cochrane de 2010 avaliou os efeitos de diferentes métodos de rastreio de DG, bem como os resultados desses métodos para as grávidas e para os recém-nascidos (grau de recomendação B e nível de evidência 3A). ${ }^{13}$ Os resultados encontrados, consistentes com revisões prévias, concluem pela escassa evidência sobre se o rastreio da DG melhora os resultados para a mãe e para o recémnascido; a evidência foi igualmente considerada insuficiente para identificar que tipos de rastreio têm impacto positivo nos resultados perinatais. Não existe igualmente evidência de que as mulheres identificadas com os novos critérios de DG, bem como os seus filhos, possam beneficiar com essa classificação mais agressiva.

\section{IMPLICAÇÕES DE DIAGNOSTICAR COMO DOENTE UMA MAIOR PROPORÇÃO DE GRÁVIDAS}

Após o diagnóstico de DG que, na maioria dos casos, corresponde ao diagnóstico de um fator de risco assintomático, o auto-conceito de saúde da mulher grávida declinará. ${ }^{20}$ Os prejuízos inerentes ao rotular como doentes ou «em risco» pessoas previamente saudáveis, mesmo que assintomáticas, têm sido progressivamente reconhecidos na literatura internacional ${ }^{21}$ e começam precisamente pelo declínio do auto-conceito de saúde que parece estar presente, paradoxalmente, nas populações com maior acesso a cuidados médicos. ${ }^{22}$ Estão igualmente descritos 
efeitos do stress materno durante a gravidez no neuro-desenvolvimento infantil. ${ }^{23} \mathrm{Um}$ diagnóstico assente apenas num fator de risco não equivale nem a doença certa nem a cura garantida se forem seguidas as intervenções propostas, pois há que ter em conta conceitos como o de «número necessário a tratar» (NNT) e de «número necessário para prejudicar» (NNH). Estes conceitos devem ser explicados aos pacientes para que possam partilhar as decisões acerca da sua saúde,$^{24}$ mas estão indisponíveis no caso dos novos critérios de diagnóstico de DG. Há ainda que reconhecer a existência de co-fatores de vária índole ignorados pela aritmética simplificadora das tabelas de risco. ${ }^{25-27}$

$\mathrm{O}$ aumento dos intervalos de valores que classificam uma pessoa como doente é uma das formas conhecidas de sobrediagnóstico. ${ }^{z} \mathrm{O}$ sobrediagnóstico é um problema mundial crescente, relacionado com o avanço tecnológico mas também com uma cultura de sobrestimação dos benefícios dos tratamentos, com práticas defensivas e com interesses económicos vários e foi já acusado de substituir o objectivo da Medicina de «curar os doentes» pelo de «adoecer os saudáveis». ${ }^{28}$

É uma recomendação do consenso da AID que a decisão sobre a aplicação da estratégia de rastreio a todas as grávidas ou apenas às consideradas de maior risco para a DG tenha em conta, em cada local, a prevalência de alterações do metabolismo glucídico. A prevalência de DG em Portugal foi estimada em $3,9 \%$, em 2009. ${ }^{29}$ Não poderemos prever qual o valor de prevalência perante os novos critérios propostos.

Recentemente, o Grupo Espanhol de Diabetes e Gravidez decidiu não adotar os novos critérios propostos enquanto estudos sobre a população local não comprovassem que as grávidas do país teriam o mesmo risco que as do estudo HAPO, se sujeitas aos mesmos critérios. ${ }^{30}$ Estes responsáveis espanhóis apontaram o mesmo motivo pelo qual já não haviam adotado os critérios da ADA em 2000 (os critérios de Carpenter e Coustan, que em Portugal foram prontamente adotados em 1998, ${ }^{31}$ vigorando até Janeiro de 2011): o resultado de um estudo prospetivo que concluiu que a mudança para os critérios de diagnóstico de DG de Carpenter e Coustan aumentaria muito a prevalência de DG em Espanha, mas que as grávidas assim diagnosticadas não teriam um aumento do risco de eventos perinatais adversos tão expressivo como o obtido no estudo de Toronto, ${ }^{32}$ que havia sustentado a adoção dos critérios da ADA $\ddagger .^{33}$

\section{IMPLICAÇÕES PRÁTICAS DO TRATAMENTO INTENSIVO DA DIABETES GESTACIONAL}

O estudo ACHOIS ${ }^{34}$ mostrou haver redução de um conjunto de eventos perinatais adversos (mas não de cada um deles isoladamente) em mulheres grávidas, entre as 24 e as 34 semanas de gestação, com metabolismo glucídico alterado, às quais se aplicou um tratamento intensivo (aconselhamento nutricional, monitorização da glicemia capilar e, se necessário, insulinoterapia).

Este estudo, realizado no Reino Unido e na Austrália, incluiu 1.000 mulheres grávidas e comparou o tratamento intensivo com o acompanhamento clássico da gravidez. Sendo o acompanhamento clássico padrão nestes países muito diferente do português, não será seguro afirmar que se venham a verificar entre nós diferenças na probabilidade de eventos perinatais adversos semelhantes às encontradas no estudo ACHOIS.

Além disso, para o estudo ACHOIS foram recrutadas de entre as grávidas com fatores de risco para DG ou com uma prova de O'Sullivan positiva, aquelas que, duas horas após a ingestão de $75 \mathrm{~g}$ de glicose, apresentassem glicemia inferior a $140 \mathrm{mg} / \mathrm{dL}$ em jejum e entre 140 e $200 \mathrm{mg} / \mathrm{dL}$. Estas mulheres tinham, portanto, critérios de diagnóstico muito diferentes (menos apertados) dos agora ditados pela DGS, pelo que não é seguro afirmar que os benefícios encontrados no ACHOIS sejam replicáveis em todas as mulheres grávidas e utilizando os novos valores de referência propostos.

Por outro lado, a redução de eventos perinatais adversos foi verificada apenas se considerados estes eventos de forma global e não de cada um deles de forma individual, sendo requerida a realização de estudos com maior potência estatística para confirmar a redução destes eventos.

Uma revisão sistemática e meta-análise sobre o tratamento da DG, publicada em 2010, ${ }^{35}$ concluiu que o tratamento hipoglicemiante apenas reduz o risco para distócia de ombros (maior redução para tratamento mais intensivo) e para macrossomia (sem relação com intensidade do tratamento), não encontrando diferenças significativas na taxa de cesarianas, no número de mortes neonatais e de traumas obstétricos nem na taxa de internamento em cuidados intensivos neonatais (grau de recomendação B e

† Glicemia igual ou superior a 95 mg/dL em jejum, a 180 mg/dL uma hora após ingestão de $100 \mathrm{~g}$ de glicose, a $155 \mathrm{mg} / \mathrm{dL}$ após duas horas e a $140 \mathrm{mg} / \mathrm{dL}$ após três horas. 
nível de evidência 3A).

Assim, podemos perceber que nem todo o aumento de eventos perinatais adversos previsto no estudo HAPO pelo aumento da glicemia durante a gestação pode ser evitado com o tratamento intensivo.

Por outro lado, estando estabelecida uma associação estatística entre eventos perinatais adversos e o aumento da glicemia capilar, está ainda por provar a respetiva relação de causalidade. Na distocia de ombros, por exemplo, embora estejam identificados fatores (como a macrossomia) que a ela predispõem, na maioria dos casos ela ocorre na ausência de fatores de risco, sendo mesmo considerada uma ocorrência maioritariamente imprevisível. ${ }^{36,37}$ Outro estudo apresenta conclusões no mesmo sentido e também que, após eliminadas as variáveis de confundimento, nem a macrossomia parece ter uma associação significativa com a distocia de ombros, ${ }^{38}$ já que $78 \%$ dos recém-nascidos grandes para a idade gestacional nascem de mães sem fatores de risco. Poderão existir explicações alternativas para o facto de estes três fatores surgirem em simultâneo nas mesmas grávidas: idade materna mais avançada, IMC prévio mais elevado e características genéticas que favoreçam não só o aparecimento de glicemias mais elevadas mas também de macrossomia fetal e distocia de ombros.

Para além da falta de evidência que sustente o benefício no tratamento intensivo de todas as mulheres com DG à luz dos novos critérios, é de salientar a ausência de dados sobre a segurança destas intervenções, tal como conclui uma revisão sistemática/meta-análise recente que considera que os riscos do tratamento da DG não foram devidamente endereçados nos ensaios disponíveis. ${ }^{35}$

\section{IMPLICAÇÕES DA MONITORIZAÇÃO DA GLICEMIA CAPILAR}

Os resultados de dois ensaios clínicos, ambos de pequena dimensão, sobre a auto-vigilância da glicemia em mulheres com DG foram contraditórios. ${ }^{39,40}$ Num deles, em 1986, ${ }^{40}$ mulheres com DG (diagnosticada por PTGO com 100 g realizada se obtivessem glicemias de $135 \mathrm{mg} / \mathrm{dL}$ ou mais após uma sobrecarga de $50 \mathrm{~g}$ oral) que monitorizaram a sua glicemia duas vezes por dia foram, comparativamente com as que não monitorizaram a glicemia, mais frequentemente insulinizadas e tiveram menos RN macrossómicos e grandes para a idade gestacional. Pelo contrário, num outro ensaio, de dimensão sobreponível, rea- lizado em 2002, em grávidas com DG ligeira (glicemias em jejum inferiores a $95 \mathrm{mg} / \mathrm{dL}$ após medidas dietéticas), a auto-monitorização da glicemia não mostrou vantagens significativas, nem no controlo glicémico, nem na taxa de eventos perinatais adversos. ${ }^{39}$ Além disso, estudos em pessoas diabéticas tipo 2 não insulino-tratadas têm falhado sistematicamente em demonstrar eficácia, mesmo no controlo de indicadores de resultado intermédios como a $\mathrm{HbA}_{1} \mathrm{C}^{41-43}$

Para além da falta de evidência acerca dos seus benefícios, têm sido apontados vários riscos associados à monitorização da glicemia capilar na população de diabéticos, nomeadamente o de depressão e o facto de o doente ter tendência a realizar de forma errada o auto-ajuste das medidas farmacológicas e não-farmacológicas, originando oscilações nefastas dos valores de glicemia. ${ }^{41-43}$ Por outro lado, os custos são elevados e não deixa de ser uma análise invasiva que provoca dor (mesmo que ligeira) e alteração da rotina diária da grávida.

\section{IMPLICAÇÕES DE OUTRAS INTERVENÇÕES INTENSIVAS}

No estudo ACHOIS ${ }^{34}$ houve um aumento de internamentos na unidade de cuidados neonatais e das induções de parto para as mulheres que tiveram um acompanhamento intensivo. Uma revisão sistemática da Cochraneem 2009 concluiu que o tratamento da DG (utilizando os critérios clássicos) aumenta o risco de parto induzido (grau de recomendação B e nível de evidência 3A). ${ }^{44}$ Estes dados suscitam a preocupação pela possibilidade de os novos critérios de DG resultarem no aumento de intervenções porventura evitáveis. O internamento na unidade de cuidados neonatais pode aumentar a ansiedade materna durante $\mathrm{o}$ internamento, perturbar a vinculação precoce mãe-filho e o estabelecimento do aleitamento materno. A indução do parto pode espelhar uma tendência para aumentar a monitorização e intervenção terapêutica que pode ser desnecessária, desadequada ou mesmo prejudicial.

\section{RECLASSIFICAÇÃO PÓS-PARTO}

Tal como nas orientações técnicas precedentes da DGS, também nesta é recomendada a realização de reclassificação do metabolismo glucídico da puérpera seis a oito semanas após o parto. A orientação recomenda que esta reclassificação seja realizada a nível da consulta hospitalar, não salientando que, nos casos em que isso não seja 
possível, ela possa ser realizada pelo médico de família.

O período pós-parto constitui uma fase de readaptação da estrutura familiar com muitas mudanças emocionais e sociais que podem favorecer $\mathrm{o}$ abandono do acompanhamento a nível hospitalar por parte da puérpera. Os cuidados de saúde primários estão não só mais disponíveis como têm maior capacidade de contacto com a família nesta fase, favorecendo o cumprimento desta tarefa importante para determinar o acompanhamento que esta mulher irá ter no futuro.

\section{IMPLICAÇÕES NOS RECURSOS DE SAÚDE}

Apesar de serem conhecidos estudos internacionais ${ }^{45} \mathrm{e}$ portugueses ${ }^{46}$ que concluíram que a instituição de medidas preventivas preconizadas por peritos pode ter um impacto insustentável nos sistemas de saúde, as novas orientações são propostas sem que seja avaliado o impacto da sua implementação.

Em Portugal, num período em que se pretende uma redução anual de $10 \%$ do orçamento da saúde e em que a capacidade instalada de alguns hospitais não permite o agendamento de primeiras consultas de Ginecologia nos tempos de resposta garantidos previstos na lei, ${ }^{47} \mathrm{O}$ aumento de custos associado ao aumento de carga de trabalho que a implementação dos novos critérios seguramente trará, faz adivinhar dificuldades acrescidas no cumprimento dos objetivos orçamentais e dos tempos de resposta garantidos. Não é possível esquecer também os custos associados à monitorização intensiva da glicemia capilar, integralmente suportados pelo SNS. Por outro lado, o desvio de recursos limitados para cumprimento destas orientações irá provavelmente penalizar a realização de outras intervenções e atividades, nomeadamente de âmbito curativo, com custos de oportunidade seguramente muito relevantes.

A preocupação sobre o impacto que os novos critérios propostos pelo consenso da AID e do Grupo de Estudos da Gravidez podem ter nos sistemas de saúde está patente em artigos publicados noutros países. ${ }^{48,49}$

Estudos de custo-efetividade realizados nos $\mathrm{EUA}^{50}(47)$ e no Canadá ${ }^{51}$ mostraram não existir evidência suficientemente convincente para recomendar o rastreio universal de DG. O National Institute of Clinical Excellence refere que apenas o rastreio seletivo orientado pela presença de fatores de risco de DG é custo-efetivo. ${ }^{52}$

Um estudo francês de 2002 mostrou que aplicar a PTGO a todas as grávidas em vez da prova de O'Sullivan quadru- plicaria os custos. ${ }^{53} \mathrm{Um}$ estudo em Itália verificou que a diferença entre rastrear todas as grávidas com prova de O'Sullivan, em comparação com o rastreio apenas das grávidas com fatores de risco de DG, originaria um custo superior em 18 por cada evento perinatal adverso evitado (424 em vez de 406$)^{.54}$

No estudo ACHOIS, ${ }^{34}$ o NNT da intervenção foi de 34, o que implica que será necessário um investimento importante de recursos materiais, humanos, organizacionais e emocionais para reduzir o risco de eventos perinatais adversos. Um estudo australiano estimou que a aplicação dos resultados do ACHOIS custaria, em 2002, cerca de 45.000 (entre despesas do sistema de saúde e das famílias), por cada 100 mulheres com DG tratada, considerando contudo os autores que tal seria um custo provavelmente justificável em países ricos..$^{55}$

\section{OUTROS ASPETOS METODOLÓGICOS}

Alguns aspetos não específicos desta Norma merecem ser considerados. Em primeiro lugar, uma orientação técnica dirigida a um largo segmento da população deve ser objecto de revisão por um painel alargado que inclua não só especialistas da área em estudo como metodólogos e generalistas. ${ }^{56}$ Em segundo lugar, deve ser testada a adequação ao cenário nacional das recomendações resultantes dos estudos usados em seu suporte. Por último, a validação de Normas através de um instrumento próprio ${ }^{57}$ é muito recomendável. Tanto quanto sabemos nenhuma destas medidas terá sido tomada em relação a esta Norma.

\section{CONCLUSÕES}

Relativamente a cada componente das recomendações da DGS, de acordo com a evidência disponível, podemos concluir que:

- A assunção do limite de normalidade nos $92 \mathrm{mg} / \mathrm{dL}$ para glicemia em jejum na primeira consulta de vigilância não é suportada pelo estudo HAPO, uma vez que este não avaliou o impacto da presença destes valores de glicemia antes das 24 semanas de gestação.

- A PTGO com 75 g de glicose não se encontra confirmada como a melhor forma de rastreio, existindo uma meta-análise que refere que devem ser ponderados os riscos potenciais da utilização de testes com maior sensibilidade diagnóstica pelo aumento de prevalência que implicam (grau de recomendação B e nível de evidência 3A). 
- A alteração dos valores de referência é baseada no estudo HAPO, um estudo observacional, com alguns problemas metodológicos (reduzida taxa de participação, possibilidade de viés de seleção, falta de ajuste dos resultados de acordo com as variáveis de confundimento, elevada taxa de cesarianas). Uma vez que o estudo HAPO estabeleceu uma associação linear entre as variáveis, foi um consenso de peritos da AID que determinou o limite a partir da probabilidade de aumento de 1,75 dos eventos perinatais adversos, o que confere à recomendação um nível de evidência baixo.

- Uma revisão sistemática e uma meta-análise mostram que o tratamento da DG (diagnosticada com os critérios clássicos) é eficaz na redução dos casos de macrossomia e distocia de ombros mas não existe evidência ainda de que os novos critérios tenham os mesmos resultados.

- Um estudo randomizado e controlado (ACHOIS) mostra aumento de alguns eventos perinatais adversos (internamento em unidades de tratamento neonatais e indução de parto) devido ao tratamento intensivo (grau de recomendação B e nível de evidência 3A).

- Três estudos em três países diferentes mostram que o rastreio de DG a todas as grávidas não é custo-eficaz.

- Não existe evidência de que as características da população portuguesa e dos serviços de saúde portugueses permitam um impacto positivo das medidas propostas em termos de ganhos em saúde, nem que essas alterações sejam custo-efetivas quando aplicadas na nossa realidade.

Se se pretende uma medicina responsável e sustentável, decisões como a de rastrear e definir critérios diagnósticos de DG implicam uma avaliação criteriosa de toda a evidência disponível, assim como considerações éticas. ${ }^{58} \mathrm{Em}$ última análise, estas são decisões de política de saúde. ${ }^{59}$

Face aos estudos disponíveis, é discutível a robustez da evidência, quer de benefício, quer de segurança, das intervenções propostas pela nova orientação técnica e pela nova versão do consenso, tanto no que diz respeito à estratégia de rastreio, como no que se refere à instituição em grávidas com valores marginais (previamente definidos como normais) de vigilância em consulta hospitalar de obstetrícia, de aconselhamento nutricional específico, de autovigilância da glicemia e de tratamento com insulina ou antidiabéticos orais.

Parece, assim, prudente a suspensão da Norma n. ${ }^{\circ}$ 7/2011 DGS, «Diagnóstico e conduta na Diabetes Gesta- cional», regressando-se ao modelo anterior de rastreio e diagnóstico de DG (ele próprio ainda não fundamentado por estudos adequados) e até que evidência robusta recomende a sua modificação.

\section{Artigo escrito ao abrigo do novo acordo ortográfico.}

\section{AGRADECIMENTOS}

Os autores agradecem aos Drs. Daniel Pinto e António Faria Vaz pelos seus comentários às versões de trabalho do presente artigo.

\section{REFERÊNCIAS BIBLIOGRÁFICAS}

1. Boavida JM, Carvalho D, Raposo JF, Silva PM, Duarte R. Norma 001/2011 Terapêutica da diabetes mellitus tipo 2: metformina. Lisboa: Direção-Geral da Saúde; 2011.

2. Pinto D, Heleno B, Gallego R, Santos I, Santiago LM, Maria V. Norma Terapêutica da Diabetes Mellitus tipo 2: metformina - uma perspectiva crítica. Acta Med Port 2011 Mar-Abr; 24 (2): 331-8.

3. Vicente L, Boavida JM. Norma 007/2011 Diagnóstico e conduta na Diabetes Gestacional. Lisboa: Direção-Geral da Saúde; 2011.

4. Dores J, Almeida MC, Vicente L, Paiva S; Sociedade Portuguesa de Endocrinologia, Diabetes e Metabolismo, Sociedade Portuguesa de Diabetologia, Sociedade Portuguesa de Obstetrícia e Medicina Materno-Fetal e Secção de Neonatologia da Sociedade Portuguesa de Pediatria. Relatório de Consenso sobre a Diabetes e Gravidez. 2011. Disponível em http://www.dgs.pt?mid=5005\&cr=19703 [acedido em 30/06/2011].

5. International Association of Diabetes and Pregnancy Study Groups Consensus Panel. Recommendations on the dagnosis and classification of hyperglycemia in pregnancy. Diabetes Care 2010 Mar; 33 (3): 676-82.

6. HAPO Study Cooperative Research Group. Hyperglycemia and adverse pregnancy outcomes. N Engl J Med 2008 May 8; 358 (19): 1991-2002.

7. American Diabetes Association. Position statement: standards of medical care in diabetes - 2012. Diabetes Care 2012 Jan; 35 Suppl 1: 11-63.

8. American College of Obstetricians and Gynecologists: ACOG Practice Bulletin. Clinical management guidelines of gestational diabetes for obstetrician-gynecologists. Obstet Gynecol 2001 Sep; 98 (3): 525-38.

9. Hoffman L, Nolan C, Wilsson JD, Oats JJ, Simmons D. Gestational diabetes mellitus-management guidelines. The Australasian Diabetes in Pregnancy Society. Med J Aust 1998 Jul 20; 169 (2): 93-7.

10. Metzger BE, Buchanan TA, Coustan DR, de Leiva A, Dunger DB, Hadden $D R$, et al. Summary and recommendations of the fifth international workshop-conference on gestational diabetes mellitus. Diabetes Care 2007 Jul; 30 Suppl 2: S251-60.

11. Oats JJ, Mclntyre HD. Revision of guidelines for the management of gestational diabetes mellitus. Med J Aust 2004 Sep 20; 181 (6): 342.

12. The Royal Australian and New Zealand College of Obstetricians and Gynaecologists. College statement: Diagnosis of gestational diabetes mellitus. Melbourne: RANZCOG; 2008.

13. Tieu J, Middleton P, McPhee AJ, Crowther CA. Screening and subsequent management for gestational diabetes for improving maternal and infant health. Cochrane Database Syst Rev 2010 Jul 7; (7): CD007222.

14. De los Santos-Garate AM, Villa-Guillen M, Villanueva-García D, Vallejos-Ruíz ML, Murguía-Peniche MT; NEOSANO's Network. Perinatal morbidity and 
mortality in late-term and post-term pregnancy: NEOSANO perinatal network's experience in Mexico. J Perinatol 2011 Dec; 31 (12): 789-93.

15. Sermer M, Naylor CD, Gare DJ, Kenshole AB, Ritchie JW, Farine D, et al. Impact of increasing carbohydrate intolerance on maternal-fetal outcomes in 3637 women without gestational diabetes. The Toronto Tri-Hospital Gestational Diabetes Project. Am J Obstet Gynecol 1995 Jul; 173 (1): $146-56$.

16. Jensen DM, Damm P, Sørensen B, Mølsted-Pedersen L, Westergaard JG, Klebe J, et al. Clinical impact of mild carbohydrate intolerance in pregnancy: a study of 2904 nondiabetic Danish women with risk fators for gestational diabetes. Am J Obstet Gynecol 2001 Aug; 185 (2): 413-9.

17. Yang $X$, Hsu-Hage B, Zhang H, Zhang C, Zhang Y, Zhang C. Women with impaired glucose tolerance during pregnancy have significantly poor pregnancy outcomes. Diabetes Care 2002 Sep; 25 (9): 1619-24.

18. Sacks DA, Greenspoon JS, Abu-Fadil S, Henry HM, Wolde-Tsadik G, Yao JF. Toward universal criteria for gestational diabetes: the 75-gram glucose tolerance test in pregnancy. Am J Obstet Gynecol 1995 Feb; 172 (2 Pt 1): 607-14.

19. American College of Obstetrics and Gynecology. Committee opinion no. 504: screening and diagnosis of gestational diabetes mellitus. Obstet Gynecol. 2011 Sep; 118 (3): 751-3.

20. Rumbold AR, Crowther CA. Women's experiences of being screened for gestational diabetes mellitus. Aust N Z J Obstet Gynaecol 2002 May; 42 (2): 131-7.

21. Westin S, Heath I. Thresholds for normal blood pressure and serum cholesterol. BMJ 2005 Jun 25; 330 (7506): 1461-2.

22. Sen A. Health: perception versus observation. BMJ 2002 Apr 13; 324 (7342): 860-1.

23. Talge NM, Neal C, Glover V. Antenatal maternal stress and long-term effects on child neurodevelopment: how and why? J Child Psychol Psychiatry 2007 Mar-Apr; 48 (3-4): 245-61.

24. Straus SE, Sackett DL. Applying evidence to the individual patient. Ann Oncol 1999 Jan; 10 (1): 29-32.

25. Albert CM, Chae CU, Rexrode KM, Manson JE, Kawachi I. Phobic anxiety and risk of coronary heart disease and sudden cardiac death among women. Circulation 2005 Feb 1; 111 (4): 480-7.

26. Dong M, Giles WH, Felitti VJ, Dube SR, Williams JE, Chapman DP, et al. Insights into causal pathways for ischemic heart disease: adverse childhood experiences study. Circulation 2004 Sep 28; 110 (13): 1761-6.

27. Feinberg AP. Epigenetics at the epicenter of modern medicine. JAMA 2008 Mar 19; 299 (11): 1345-50.

28. Moynihan R, Doust J, Henry D. Preventing overdiagnosis: how to stop harming the ealthy. BMJ 2012 May 28; 344: e3502.

29. Observatório Nacional da Diabetes. Diabetes Factos e Números 2010. Relatório anual do Observatório Nacional da Diabetes - Portugal 2010. Disponível

em: http://www.spd.pt/images/relatorioanualdiabetes\%20fev\%202011.pdf [acedido em 30/06/2011].

30. Corcoy R, Lumbreras B, Bartha JL, Ricart W; Grupo Español de Diabetes y Embarazo. Nuevos critérios diagnósticos de diabetes mellitus gestacional a partir del estudio HAPO. ¿Son válidos en nuestro medio? Gac Sanit 2010 Jul-Ago; 24 (4): 361-3.

31. Dinis JA, Calado B. Diabetes e Gravidez. Circular Normativa nº 8 - Divisão de Doenças Genéticas, Crónicas e Geriátricas. Lisboa: Direção-Geral da Saúde; 1998.

32. Naylor CD, Sermer M, Chen E, Farine D. Selective screening of gestational diabetes mellitus. N Engl J Med 1997 Nov 27; 337 (22): 1591-6.

33. Ricart W, López J, Mozas J, Pericot A, Sancho MA, González N, et al. Potential impact of American Diabetes Association (2000) criteria for diagnosis of gestational diabetes mellitus in Spain. Diabetologia 2005 Jun; 48 (6): 1135-41.

34. Crowther CA, Hiller JE, Moss JR, McPhee AJ, Jeffries WS, Robinson JS. Effect of treatment of gestational diabetes mellitus on pregnancy outcomes. N Engl J Med 2005 Jun 16; 352 (24): 2477-86.

35. Horvath K, Koch K, Jeitler K, Matyas E, Bender R, Bastian H, et al. Effects of treatment in women with gestational diabetes mellitus: systematic review and meta-analysis. BMJ 2010 Apr 1; 340: c1395.

36. Baskett TF, Allen AC. Perinatal implications of shoulder dystocia. Obstet Gynecol 1995 Jul; 86 (1): 14-7.

37. Gottlieb AG, Galan HL. Shoulder dystocia: an update. Obstet Gynecol Clin North Am 2007 Sep; 34 (3): 501-31.

38. Mehta SH, Blackwell SC, Bujold E, Sokol RJ. What factors are associated with neonatal injury following shoulder dystocia? J Perinatol 2006 Feb; 26 (2): 85-8.

39. Homko CJ, Sivan E, Reece EA. The impact of self-monitoring of blood glucose on self-efficacy and pregnancy outcomes in women with diet-controlled gestational diabetes. Diabetes Educ 2002 May-Jun; 28 (3): 43543.

40. Goldberg JD, Franklin B, Lasser D, Jornsay DL, Hausknecht RU, GinsbergFellner $F$, et al. Gestational diabetes: impact of home glucose monitoring on neonatal birthweight. Am J Obstet Gynecol 1986 Mar; 154 (3): 54650.

41. O'Kane MJ, Bunting B, Copeland M, Coates VE; ESMON Study Group. Efficacy of self monitoring of blood glucose in patients with newly diagnosed type 2 diabetes (ESMON study): randomised controlled trial. BMJ 2008 May 24; 336 (7654): 1174-7.

42. Farmer AJ, Wade AN, French DP, Simon J, Yudkin P, Gray A, et al. Blood glucose self-monitoring in type 2 diabetes: a randomised controlled trial. Health Technol Assess 2009 Feb; 13 (15): iii-iv, ix-xi, 1-50.

43. Simon J, Gray A, Clarke P, Wade A, Neil A, Farmer A; Diabetes Glycaemic Education and Monitoring Trial Group. Cost effectiveness of self monitoring of blood glucose in patients with non-insulin treated type 2 diabetes: economic evaluation of data from the DiGEM trial. BMJ 2008 May 24; 336 (7654): 1177-80.

44. Alwan N, Tufnell DJ, West J. Treatments for gestational diabetes. Cochrane Database Syst Rev 2009 Jul 8; (3): CD003395.

45. Yarnall KS, Pollak KI, Østbye T, Krause KM, Michener JL. Primary care: is there enough time for prevention? Am J Public Health 2003 Apr; 93 (4): 635-41.

46. Pinto D, Corte-Real S, Nunes JM. Actividades preventivas - quanto tempo sobra? Rev Port Clin Geral 2010 Set-Out; 26 (5): 455-64.

47. Observatório Português de Sistemas de Saúde. Relatório de primavera 2010. Disponível em: http://www.observaport.org/rp [acedido em 30/06/2011].

48. Moses RG. New Consensus Criteria for GDM Problem solved or a Pandora's box? Diabetes Care 2010 Mar; 33 (3): 690-1.

49. Ryan EA. Diagnosing gestational diabetes. Diabetologia 2011 Mar; 54 (3): 480-6. 
50. U.S. Preventive Services Task Force. Screening for gestational diabetes mellitus: U.S. Preventive Services Task Force recommendation statement. Ann Intern Med 2008 May 20; 148 (10): 759-65.

51. Canadian Task Force on the Periodic Health Examination. The Canadian Guide to Clinical Preventive Health Care. Ottawa: Health Canada 1994. p. $15-23$.

52. National Collaborating Centre for Women's and Children's Health. Diabetes in Pregnancy: management of diabetes and its complications from preconception to the postnatal period. London: RCOG Press; 2008.

53. Poncet B, Touzet S, Rocher L, Berland M, Orgiazzi J, Colin C. Cost-effectiveness analysis of gestational diabetes mellitus screening in France. Eur J Obstet Gynecol Reprod Biol 2002 Jul 10; 103 (2): 122-9.

54. Di Cianni G, Volpe L, Casadidio I, Bottone P, Marselli L, Lencioni C, et al. Universal screening and intensive metabolic management of gestational diabetes: cost-effectiveness in Italy. Acta Diabetol 2002 Jun; 39 (2): 6973.

55. Moss JR, Crowther CA, Hiller JE, Willson KJ, Robinson JS; Australian Carbohydrate Intolerance Study in Pregnant Women Group. Costs and consequences of treatment for mild gestational diabetes mellitus - evaluation from the ACHOIS randomised trial. BMC Pregnancy Childbirth 2007 Oct 28; 7:27.

56. Lim W, Arnold DM, Bachanova V, Haspel RL, Rosovsky RP, Shustov AR, et al. Evidence-based guidelines - an introduction. Hematology Am Soc Hematol Educ Program 2008: 26-30.

57. AGREE Collaboration. Development and validation of an international ap- praisal instrument for assessing the quality of clinical practice guidelines: the AGREE project. Qual Saf Health Care 2003 Feb; 12 (1): 18-23.

58. Gets L, Kirkengen AL, Hetlevik I, Sigurdsson JA. Individually based preventive medical recommendations - are they sustainable and responsible? A call for ethical reflection. Scand J Prim Health Care 2005 Jun; 23 (2): 65-7.

59. Heath I, Mangin D, Toop L, Brodersen J. The future of national health systems. Br J Gen Pract. 2011 May; 61 (586): 319-20.

\section{CONFLITO DE INTERESSES}

Os autores declaram não existirem conflitos de interesses relacionados com o conteúdo do artigo. Mónica Granja é editora da Revista Portuguesa de Medicina Geral e Familiar, não tendo participado no processo editorial deste artigo.

\author{
ENDEREÇO PARA CORRESPONDÊNCIA \\ Carla Gouveia \\ USF LoureSaudável \\ Rua Angra do Heroísmo, SN \\ 2670-487 LOURES \\ E-mail: carlapggouveia@hotmail.com
}

Recebido em 19/03/2012

Aceite para publicação em 08/06/2012

\section{ABSTRACT}

\section{NEW GUIDELINES FOR THE DIAGNOSIS AND MANAGEMENT OF GESTATIONAL DIABETES FROM THE DIRECTORATE GENERAL OF HEALTH: A CRITICAL REVIEW}

The new guidelines from the Portuguese Directorate General of Health (DGH) for gestational diabetes (GD) recommend changes in the screening, treatment and follow-up of GD. We discuss the scientific basis for these guidelines and their potential implications for improvements in health, costs, the use of health service resources and their potential impact on the newly diagnosed population of pregnant women.

No references cited by the DGH support the diagnosis of GD based on fasting plasma glucose levels above $92 \mathrm{mg} / \mathrm{dL}$ at the first prenatal appointment.

Using the new cutoff point for the diagnosis of GD for an oral glucose tolerance test performed between 24 and 28 weeks pregnancy results in a tenfold increase in the prevalence of $G D$, leading to increased allocation of health services resources for management and follow up.

A review of the literature reveals no benefit from universal prenatal screening as opposed to selective screening of pregnant women with known risk factors (grade of Recommendation B and level of evidence $3 \mathrm{~A}$ ). Cost-effectiveness studies also favour selective screening over universal screening.

Treatment of women newly diagnosed with GD has not shown a reduction in perinatal morbidity, as predicted by the HAPO study. Potential adverse effects of this approach have not been assessed.

It would probably be wise to keep the new guideline on hold and return to the previous protocol of GD screening until more robust evidence supports changes.

Key-words: Diabetes; Gestational; Screening; Diagnosis; Disease Management; Clinical Protocols. 